\title{
Apego Materno-Fetal e a Percepção Materna acerca da Capacidade Interativa do Bebê no Primeiro Mês
}

\author{
Patrícia Alvarenga \\ Janaína Nascimento Teixeira \\ Aline Carvalho Peixoto \\ Universidade Federal da Bahia \\ Salvador, BA, Brasil
}

\begin{abstract}
RESUMO
A percepção da mãe acerca das capacidades interativas do recém-nascido tem sido apontada como um fator crucial para o estabelecimento de padrões interativos de qualidade entre a díade mãe-filho. O objetivo deste estudo foi examinar as relações entre o apego materno-fetal e a percepção da mãe acerca das capacidades interativas do bebê. Participaram do estudo 64 mães de bebês do sexo masculino, que responderam a Escala de Apego Materno-fetal, no terceiro trimestre da gestação, e uma entrevista sobre a experiência da maternidade, no primeiro mês do bebê. Houve uma correlação positiva entre o apego materno-fetal e a percepção geral das capacidades interativas do bebê $(r=0,23 ; p<0,05)$. A análise de regressão confirmou que a escolaridade materna e o apego materno-fetal explicaram $12 \%$ da variância na percepção da mãe acerca das capacidades interativas dos bebês. Discute-se a relevância da relação da mãe com o feto durante a gestação para a interação mãe-bebê no período pós-natal.
\end{abstract}

Palavras-chave: Capacidades Interativas; Maternidade; Apego Materno-Fetal.

\section{ABSTRACT}

\section{Maternal-Fetal Attachment and Maternal Perception about Infant Interactive Skills in the First Month}

The mother's perception about the infant's interactive skills has been identified as a crucial factor for the establishment of appropriate interactive patterns between the mother-child dyad. The aim of this study was to examine the relations between maternal-fetal attachment and the mother's perception about the infant's interactive skills. The study included 64 mothers of boys who responded the Maternal-fetal Attachment Scale in the third trimester of pregnancy, and an interview about the experience of motherhood, during the infant's first month of life. There was a positive correlation between maternal-fetal attachment and the general perception of the infant interactive skills $(r=0,23 ; \mathrm{p}<0,05)$. Regression analysis confirmed that maternal education and maternal-fetal attachment explained $12 \%$ of variance in the mother's perception of the infant's interactive skills. The relevance of the relationship between mother and fetus during pregnancy for mother-infant interaction in the postnatal period is discussed.

Keywords: Interactive Skills; Maternity; Maternal-Fetal Attachment.

\section{RESUMEN}

Apego Materno-Fetal y la Percepción Materna sobre la Capacidad Interactiva del Bebé en el Primer Mes

La percepción de la madre acerca de las capacidades interactivas del recién nacido ha sido identificada como un factor crucial para el establecimiento de patrones interactivos de calidad entre la díada madre-hijo. El objetivo del estudio fue examinar la relación entre el apego materno-fetal y la percepción de la madre acerca de las capacidades interactivas del bebé. El estudio incluyó a 64 madres de niños varones que respondieron a la Escala de Apego Materno-Fetal en el tercer trimestre del embarazo, y a una entrevista sobre la experiencia de la maternidad, en el primer mes de vida. Hubo una correlación positiva entre el apego materno-fetal y la percepción general de las capacidades interactivas del bebé $(\mathrm{r}=0,23, \mathrm{p}<0,05)$. El análisis de regresión confirmó que la escolaridad materna y el apego materno-fetal explicaron $12 \%$ de la varianza en la percepción de la madre acerca de las capacidades interactivas de los bebés. Se discute la importancia de la relación entre la madre y el feto durante el embarazo para la interacción madre-hijo en el período postnatal.

Palabras clave: Capacidades Interactivas; Maternidad; Apego Materno-Fetal. 
Nos últimos quarenta anos a literatura tem acumulado evidências acerca da importância dos padrões de interação e da qualidade da relação mãe-criança ao longo do primeiro ano de vida para o desenvolvimento infantil (Fraley, Roisman, \& Haltigan, 2013; Vandell, Belsky, Burchinal, Steinberg, \& Vandergrift, 2010). Padrões interativos caracterizados pela sincronia entre os comportamentos dos dois membros da díade e pela responsividade sensível da mãe (Van den Boom, 1994) estão relacionados ao desenvolvimento de um perfil de apego seguro e a melhores resultados desenvolvimentais para a criança (Cerezo, Trenado, \& Pons-Salvador, 2012; Fearon, Bakermans-Kranenburg, van IJzendoorn, Lapsley, \& Roisman, 2010).

O estudo do impacto das características físicas e do perfil interativo do bebê sobre os padrões de interação que se estabelecem entre a díade ganhou importância ao longo dos anos, e a noção de que tais fatores afetam de modo significativo a interação mãe-bebê é consensual (Aquino \& Salomão, 2011; Klaus \& Kennel, 1992). Bowlby (2002) destacou o potencial inato do recémnascido para despertar a atenção e atrair os cuidados da mãe ou de outros adultos através de comportamentos que fazem parte do sistema de apego, como o choro, o sorriso e a emissão de sons ou vocalizações. O autor também afirmou que os bebês apresentam certo nível de discriminação dos estímulos do ambiente desde o seu nascimento, assim como uma tendência a responder de maneira diferenciada a estímulos proporcionados por seres humanos como a voz, o rosto humano, e os estímulos táteis e cinestésicos.

Além dessas características inatas que seriam comuns aos indivíduos da espécie humana, a literatura também aponta para diferenças individuais nos recémnascidos, que podem produzir impacto na interação mãe-bebê. Por exemplo, o estudo de Loo, Ohgi, Howard, Tyler e Hirose (2005), com uma amostra japonesa, revelou que menor irritabilidade, maior estabilidade na coloração da pele, e menor nível de tremores no recém-nascido estiveram relacionados a comportamentos de cuidado mais frequentes na mãe no primeiro mês de vida. Além disso, o peso ao nascer e características da criança como o pico de excitação e a irritabilidade explicaram $31 \%$ da variância no comportamento da mãe durante a interação no primeiro mês de vida. Um estudo semelhante realizado por Zhu, Loo, Min, Yin, Luo, Chen, et al. (2007) com uma amostra chinesa indicou que o pico de excitação e os sobressaltos do bebê explicaram 34\% da variância no comportamento materno durante a interação no primeiro mês de vida. Outro estudo recente comparou o impacto de diferentes perfis comportamentais de bebês, avaliados na oitava semana de vida, sobre a interação da díade na décima segunda semana. Os resultados revelaram que a qualidade da interação mãe-bebê foi menor no grupo de díades com bebês de perfil retraído, quando comparada à qualidade de interação em díades com bebês de perfil extrovertido ou de baixa ativação. Entre os dois últimos grupos citados, houve uma leve tendência a maior qualidade de interação no grupo de bebês extrovertidos (Costa \& Figueiredo, 2012).

A partir do reconhecimento do papel ativo do bebê, alguns autores chamaram a atenção para o fato de que o aumento da atenção dos pais às competências interativas dos seus filhos pode melhorar a interação pais-bebê (Brazelton, 1988; Klaus \& Kennel, 1992). Quando as mães estão mais familiarizadas ou atentas às competências do recém-nascido, se tornam mais propensas a estimular a criança, prestar atenção nos seus comportamentos e serem mais responsivas aos sinais do bebê. O estudo experimental conduzido por Wendland-Carro, Piccinini e Millar (1999) revelou que díades cujas mães foram submetidas a uma intervenção que visou a demonstrar as capacidades interativas do recém-nascido, apresentaram maior frequência de sequências interativas com co-ocorrência de verbalizações, olhares e contato físico, quando comparadas às díades que foram submetidas a uma intervenção que focalizava cuidados de saúde e higiene com o bebê.

Outros pressupostos importantes relacionados aos padrões de interação mãe-criança no primeiro ano de vida referem-se à noção de que a vinculação emocional e a relação da mãe com o bebê têm início ainda no período pré-natal, e que o processo de transição para a maternidade que ocorre ao longo da gestação, também tem um impacto importante sobre a relação que a díade estabelecerá após o nascimento (Klaus \& Kennel, 1992; Piccinini, Gomes, Nardi, \& Lopes, 2008). Nesse contexto, o conceito de apego maternofetal, definido como o grau em que mulheres se engajam em comportamentos indicadores de afiliação e interação com o bebê durante a gravidez (Cranley, 1981), tem sido investigado como um possível preditor das características da interação mãe-criança nos primeiros meses de vida. $\mathrm{O}$ apego materno-fetal pode ser avaliado a partir de três diferentes dimensões: a afetiva (prazer sentido pela mãe ao pensar e interagir com o feto), a altruística (comportamentos que visam a proteger e preparar o bebê para o nascimento), e a cognitiva (fantasias e representações da mãe acerca das características do bebê) (Shieh, Kravitz, \& Wang, 2001).

Alguns estudos que avaliaram diretamente a interação mãe-criança por meio de métodos observacionais mostraram que o apego maternofetal esteve relacionado à responsividade materna 
(Alvarenga, Dazzani, Alfaya, Lordelo, \& Piccinini, 2013; Shin, Park, \& Kim, 2006), ou a outras variáveis ligadas ao comportamento da mãe nos períodos pré ou pós-natal, como as práticas saudáveis durante a gestação (Alhusen, Gross, Hayat, Woods, \& Sharps, 2012), a percepção de competência no papel materno (Mercer \& Ferketich, 1994); o apego precoce da mãe com o bebê no período pós-parto (Shin, Park, \& Kang 2004) e comportamentos emocionais direcionados ao recém-nascido (Taffazoli, Asadi, Aminyazdi \& Shakeri, 2015). Com a percepção dos movimentos do feto e a realização de ultrassonografias, especialmente a partir do terceiro trimestre da gestação, o apego materno-fetal tende a intensificar-se (Piccinini et al., 2008; Yarcheski, Mahon, Yarcheski, Hanks, \& Cannella, 2009). Além disso, fatores como maior nível de escolaridade, menor número de filhos e o suporte dado à gestante durante o período da gravidez, com destaque para a figura do pai do bebê, favorecem o apego materno-fetal (Alhusen, 2008; Alvarenga, Dazzani, Alfaya, Lordelo, \& Piccinini, 2012; Pisoni et al., 2014).

A literatura indica o impacto dos processos psicológicos que ocorrem durante a gestação, período em que a mulher se prepara para a maternidade, sobre a interação mãe-bebê. Nesse contexto, o conceito de apego materno-fetal tem se destacado como um importante preditor do comportamento materno e da qualidade da relação que se estabelecerá entre a díade no período pós-natal. Além das evidências quanto às relações entre o apego materno-fetal e a interação precoce entre mãe e bebê, há também estudos que indicam que quando a mãe está atenta e conhece o potencial interativo do recém-nascido, os padrões de interação que se estabelecem entre eles tendem a apresentar maior qualidade. Assim, o presente estudo investigou as relações entre o apego materno-fetal e a percepção da mãe acerca das capacidades interativas do bebê no primeiro mês de vida. A hipótese foi de que quanto maior o nível de apego maternofetal, mais frequentes seriam os indicadores da percepção da mãe acerca das capacidades interativas do bebê.

\section{MÉTODO}

\section{Participantes}

Participaram do estudo 64 mães selecionadas por conveniência, durante o período da gestação, em quatro maternidades públicas de Salvador/Bahia. Os bebês, nascidos a termo e com boas condições de saúde, eram todos do sexo masculino porque faziam parte de um estudo longitudinal que investigou preditores do comportamento antissocial na infância. Como a literatura indica associação entre o sexo masculino e o comportamento antissocial (Emerich, Rocha, Silvares, \& Gonçalves, 2012), a amostra envolveu apenas meninos.

As gestantes tinham idades que variaram entre 19 e 39 anos $(M=27,81 ; D P=5,45)$ e estavam no terceiro trimestre de gestação no momento da primeira etapa da coleta de dados, quando foram obtidas as informações sociodemográficas. A escolaridade da gestante $(M=10,78 ; \mathrm{DP}=2,28)$, em anos de estudo, indica que a maior parte da amostra tinha ensino médio incompleto ou completo. A maioria das gestantes não trabalhava fora $(64,1 \%)$ e a renda familiar média foi de $\mathrm{R} \$ 834,69(\mathrm{DP}=\mathrm{R} \$ 557,24)$, equivalentes a 1,23 salários mínimos. A maior parte das gestantes residia com o pai do bebê $(87,5 \%)$ e o número médio de filhos da gestante foi de $0,83(\mathrm{DP}=0,99)$.

Com relação à gravidez, a maioria das participantes não estava na sua primeira gestação $(57,8 \%)$, teve uma gravidez inesperada $(62,5 \%)$ e sem complicações $(79,7 \%)$. A maioria das gestantes não tinha problemas de saúde $(85,9 \%)$ e teve parto cesáreo $(54 \%)$, aproximadamente, na quadragésima semana de gestação $(\mathrm{M}=39,4 ; \mathrm{DP}=1,89)$. Quanto aos bebês, o peso médio no momento do nascimento foi de $3.453,36 \mathrm{~g}$ $(\mathrm{DP}=463,28 \mathrm{~g})$ e o comprimento médio foi de 48,48 $\mathrm{cm}(\mathrm{DP}=8,23 \mathrm{~cm})$. A maioria dos bebês $(75 \%)$ não precisou de internação hospitalar no primeiro mês de vida. A Tabela 1 apresenta as características da amostra. É importante salientar que em algumas das variáveis analisadas o número de participantes é inferior a 64 porque algumas gestantes não souberam relatar certas informações, tais como o número de semanas de gestação, a renda familiar e o comprimento do bebê ao nascer.

\section{Delineamento e Procedimento}

Foi utilizado um delineamento correlacional (Dancey \& Reidy, 2013), envolvendo, como variável preditora, o apego materno-fetal, e como variável predita, a percepção materna acerca das capacidades interativas do bebê. As participantes foram contatadas e convidadas a participar da pesquisa nos hospitais onde faziam o pré-natal. O primeiro contato ocorreu no terceiro trimestre da gestação, quando as participantes responderam individualmente a Ficha de Dados Sociodemográficos e Saúde da Gestante, e a Escala de Apego Materno-fetal. Quando os bebês completaram um mês de vida, as participantes responderam a Entrevista Semiestruturada sobre a Experiência da Maternidade e a Ficha de Saúde do Bebê. O estudo foi aprovado pelo Comitê de Ética da Faculdade de Filosofia e Ciências Humanas da Universidade Federal da Bahia. 
TABELA 1

Dados Sociodemográficos, Informações sobre a gestação e Características dos bebês

\begin{tabular}{|c|c|c|c|c|c|}
\hline \multirow{2}{*}{ Variáveis } & \multirow{2}{*}{ Niveis } & \multicolumn{4}{|c|}{ Valores } \\
\hline & & $n$ & $\%$ & $M$ & $D P$ \\
\hline Idade da gestante & & 64 & & 27,81 & 5,45 \\
\hline Escolaridade da gestante (em anos) & & 64 & & 10,78 & 2,28 \\
\hline \multirow[t]{2}{*}{ Ocupação da gestante } & Trabalham & 23 & 35,9 & & \\
\hline & Não trabalham & 41 & 64,1 & & \\
\hline Renda familiar & & 61 & & $\mathrm{R} \$ 834,69$ & $\mathrm{R} \$ 557,24$ \\
\hline Número de filhos & & 64 & & 0,83 & 0,99 \\
\hline \multirow[t]{2}{*}{ A gestante vive com o pai do bebê? } & Sim & 56 & 87,5 & & \\
\hline & Não & 08 & 12,5 & & \\
\hline \multirow[t]{2}{*}{ Primeira gravidez? } & Sim & 27 & 42,2 & & \\
\hline & Não & 37 & 57,8 & & \\
\hline \multirow[t]{2}{*}{ Gravidez esperada? } & Sim & 24 & 37,5 & & \\
\hline & Não & 40 & 62,5 & & \\
\hline \multirow[t]{2}{*}{ Complicação na gravidez? } & Sim & 13 & 20,3 & & \\
\hline & Não & 51 & 79,7 & & \\
\hline \multirow[t]{2}{*}{ Problemas de saúde da gestante? } & Sim & 09 & 14,1 & & \\
\hline & Não & 55 & 85,9 & & \\
\hline \multirow[t]{2}{*}{ Tipo de parto } & Normal & 29 & 46 & & \\
\hline & Cesáreo & 34 & 54 & & \\
\hline Número de semanas de gestação & & 62 & & 39,4 & 1,89 \\
\hline Peso do bebê ao nascer ( $\mathrm{em} \mathrm{g}$ ) & & 64 & & 3453,36 & 463,28 \\
\hline Comprimento do bebê ao nascer $(\mathrm{em} \mathrm{cm})$ & & 54 & & 48,48 & 8,23 \\
\hline \multirow[t]{2}{*}{ Necessidade de internação hospitalar do recém-nascido? } & Sim & 15 & 23,4 & & \\
\hline & Não & 48 & 75 & & \\
\hline
\end{tabular}

\section{Instrumentos}

Ficha de Dados Sociodemográficos e Saúde da Gestante: corresponde a um questionário criado pelos pesquisadores que investigou dados como idade da gestante, escolaridade, profissão, estado civil, existência de outros filhos, estado de saúde durante a gestação e data prevista para o nascimento do bebê.

Escala de Apego Materno-fetal: é um instrumento elaborado por Cranley (1981), que foi traduzido e validado para a população brasileira por Feijó (1999), com o objetivo de investigar o vínculo da gestante com o feto. $\mathrm{O}$ instrumento consta de 24 itens, divididos em cinco subescalas que representam diferentes aspectos da relação da mãe com o bebê. São exemplos de itens da escala: "Eu realmente estou ansiosa para ver como vai ser o meu bebê", "Eu converso com meu bebê", "Parece que meu bebê chuta e se mexe para me dizer que é hora de comer", "Eu deixo de fazer certas coisas, para o bem do meu bebê" e "Eu me imagino alimentando/amamentando o bebê". Para cada um dos itens, são oferecidas cinco possibilidades de respostas: nunca, quase nunca, em dúvida, às vezes e sempre, cuja pontuação varia de 1 a 5 , respectivamente. O escore total da escala é obtido com a soma da pontuação de cada item e pode ser classificada como: apego mínimo corresponde aos escores de 0 a 47 pontos; apego médio, escores de 48 a 71 pontos; e apego máximo, escores de 72 a 120. O índice de consistência interna (alpha de Cronbach) da escala original total é 0,85 ; 
e o da validação brasileira foi 0,63 (Feijó, 1999). A consistência interna e a validade de cada subescala isoladamente têm sido questionadas na literatura (Doan, Cox, \& Zimerman, 2003) e, por esta razão não foram utilizadas no presente estudo.

Ficha de Saúde do Bebê: foi utilizada para avaliar as condições gerais de saúde do bebê ao nascer e investigar a existência enfermidades graves ou crônicas. Além destas informações, a ficha investigou possíveis intercorrências nos últimos meses da gestação, e as condições do parto.

Entrevista Semiestruturada sobre a Experiência da Maternidade: consistiu em uma adaptação da Entrevista sobre a Maternidade e o Desenvolvimento do Segundo Filho aos Doze Meses do Núcleo de Infância e Família (2006). Esta entrevista foi utilizada para investigar as impressões e sentimentos da mãe sobre a sua relação com o recém-nascido, e abordou questões sobre a percepção da mãe sobre o bebê e sobre ela mesma como mãe, a rotina e os cuidados diários com o bebê, entre outros aspectos. Para o presente estudo, foram analisadas as respostas das mães sobre questões relacionadas ao dia-a-dia e aos cuidados com a criança, ao temperamento e à condição atual do bebê, bem como aos seus comportamentos durante o banho, a troca de fraldas, e a amamentação/alimentação. Analisouse ainda a sua percepção acerca do que agradava e desagradava o bebê, e dos comportamentos do bebê que chamavam atenção, agradavam e desagradavam a mãe. São exemplos de perguntas da entrevista: "Que tipo de coisas ele faz que te agradam?" e "Que coisas você menos gosta de fazer com ele?".

As entrevistas foram submetidas à análise de conteúdo (Bardin, 1979/2008), e os relatos das mães relacionados às questões mencionadas anteriormente, que constituíram o foco da análise do presente estudo, foram agrupados em uma grande categoria, baseada nas propostas de Schwengber e Piccinini (2005) e Wendland e Piccinini (1998), denominada capacidade interativa geral do bebê. Essa categoria inclui todos os relatos maternos acerca do potencial interativo do seu bebê. As mães descreveram diferentes comportamentos dos bebês como ouvir, vocalizar, sorrir, estar alerta, movimentar-se, mostrar agrado e desagrado, e reconhecer a mãe. Assim, essas diferentes capacidades interativas infantis descritas pelas mães foram agrupadas em quatro subcategorias, que compõem a categoria mais ampla denominada capacidade interativa geral do bebê. Assim foram denominadas e definidas as quatro subcategorias: (a) capacidade de ouvir, vocalizar e sorrir: refere-se às capacidades comunicativas do bebê, como prestar atenção ao ouvir vozes ou sons, emitir sons, resmungar e "conversar", e também à capacidade de sorrir ou olhar detidamente para a mãe. São exemplos de relatos de mães categorizados nessa subcategoria: “(...) ai por alguns minutinhos ele para de chorar como se tivesse captando a minha voz" e "(...) bem humorado dá risada demais, brinca muito e é isso!"; (b) capacidade de estar alerta e movimentar-se: inclui relatos maternos que se referem à capacidade do bebê de se manter atento aos objetos e eventos do ambiente, através do olhar e da audição, e também contempla referências à atividade motora do bebê e ao rápido desenvolvimento físico da criança com relação a este aspecto. São exemplos de relatos de mães categorizados nessa subcategoria: “(...) ela fica balançando, balançando, movimentando." e "(...) ele olha, fixa a pessoa, ele acompanha o andar da pessoa (...)"; (c) capacidade de demonstrar agrado: refere-se à percepção da mãe quanto à capacidade do bebê de demonstrar agrado em diferentes situações. As mães descreveram estímulos ou situações que julgavam agradar o bebê. São exemplos de relatos de mães categorizados nessa subcategoria: "(...) ele adora que a gente fique com ele no colo, ele adora, adora... mamar, né?" " "(...) ele gosta muito que o pai brinque muito com ele"; e (d) capacidade de demonstrar desagrado: referese à percepção da mãe quanto à capacidade do bebê de demonstrar desagrado em diferentes situações. As mães descreveram estímulos ou situações que julgavam desagradar o bebê. São exemplos de relatos de mães categorizados nessa subcategoria: "(...) eu já notei que ele não gosta de ficar sem roupa, ele chora muito" e "(...) ele chora para levantar, ele quer que eu tire ele do quarto(...)”. A análise de conteúdo foi realizada por dois codificadores independentes que receberam extenso treinamento (20 horas). O coeficiente Kappa obtido foi de 0,82 . Após a classificação das unidades de análise nas categorias descritas, a frequência de cada uma das categorias e subcategorias foi calculada e esses valores foram utilizados nas análises estatísticas.

\section{Análise de dados}

Foram utilizados procedimentos de estatística descritiva e correlacional, a partir dos dados quantitativos gerados pela análise de conteúdo das entrevistas e dos escores da escala de Apego Maternofetal. Assim, foi realizada a análise das correlações de Pearson entre as diferentes categorias dos relatos maternos, os escores totais da escala de Apego Maternofetal, e os dados sociodemográficos, as informações sobre a gestação e as características dos bebês. $\mathrm{Na}$ segunda etapa da análise, a variável preditora (escore de apego materno-fetal) e a variável predita (percepção materna acerca das capacidades interativas do bebê) foram inseridas em uma análise de regressão linear 
múltipla, utilizando a técnica stepwise, recomendada para estudos exploratórios, cujo modelo teórico e os achados empíricos ainda não são consistentes (Abbad $\&$ Torres, 2002).

\section{RESULTADOS}

Inicialmente, foram calculados os valores mínimos e máximos, as médias, e os desvios-padrão dos escores da Escala de Apego Materno-fetal e das categorias da entrevista. O escore da Escala de Apego Materno-fetal variou entre 73 e $108(\mathrm{M}=90,70 ; \mathrm{DP}=8,11)$. De acordo com os critérios estabelecidos pela própria escala, a média indica um alto nível de vinculação entre mãe e feto na amostra.

Quanto à entrevista, a média de relatos da capacidade interativa geral foi de 7,83 $(\mathrm{DP}=4,88)$. Quanto a suas subcategorias, as que apresentaram as maiores médias foram: capacidade de demonstrar desagrado $(\mathrm{M}=2,73 ; \mathrm{DP}=2,31)$ e capacidade de demonstrar agrado $(\mathrm{M}=2,52 ; \mathrm{DP}=1,57)$. As demais subcategorias apresentaram as seguintes médias: capacidade de ouvir vocalizar e sorrir $(M=1,33 ; \mathrm{DP}=1,21)$, e capacidade de estar alerta e movimentar-se $(\mathrm{M}=1,25 ; \mathrm{DP}=1,73)$.

Foi realizada uma análise das correlações (teste de correlação de Pearson) entre as frequências médias das categorias e subcategorias da entrevista, o escore na escala de Apego Materno-fetal e as informações da Ficha de Dados Sociodemográficos e Saúde da Gestante e da Ficha de Saúde do Bebê. A Tabela 2 apresenta os resultados dessas correlações. Houve correlações entre o Apego Materno-fetal e as seguintes categorias/ subcategorias da entrevista: capacidade interativa geral $(\mathrm{r}=0,23 ; \mathrm{p}<0,05)$, capacidade de ouvir, vocalizar e sorrir $(\mathrm{r}=0,23 ; \mathrm{p}<0,05)$ e capacidade de demonstrar agrado $(r=0,26 ; p<0,05)$. Essas correlações positivas indicam que quanto maior o apego da mãe com o feto durante a gestação, maior a percepção da mãe acerca dessas capacidades interativas do bebê.

Algumas variáveis sociodemográficas também estiveram correlacionadas com as categorias da entrevista. A escolaridade materna correlacionou-se positivamente com as seguintes categorias: capacidade de estar alerta e movimentar-se $(r=0,41$; $\mathrm{p}<0,001)$, capacidade de demonstrar desagrado $(\mathrm{r}=0,28, \mathrm{p}<0,05)$ e capacidade interativa geral $(\mathrm{r}=0,32 ; \mathrm{p}<0,01)$. A renda familiar correlacionou-se positivamente com as seguintes categorias: capacidade de demonstrar desagrado $(\mathrm{r}=0,31, \mathrm{p}<0,01)$ e capacidade interativa geral $(r=0,23 ; p<0,05)$. O número de filhos correlacionou-se negativamente com a categoria capacidade de demonstrar desagrado $(r=-0,25 ; p<0,05)$. A idade gestacional correlacionouse positivamente com a capacidade de ouvir, vocalizar e sorrir $(r=0,22 ; p<0,05)$. Por fim, o comprimento do bebê ao nascer correlacionou-se positivamente com a categoria capacidade de estar alerta e movimentarse $(r=0,26 ; p<0,05)$. Essas correlações indicam que quanto maior a escolaridade materna, maior renda, maior a idade gestacional, e maior o comprimento do bebê ao nascer, mais frequentes são também os indicadores da percepção da mãe acerca das capacidades interativas do bebê. Por outro lado, quanto menor o número de filhos, mais frequentes são esses indicadores.

TABELA 2

Correlações de Pearson entre Apego Materno-fetal, Categorias da Entrevista e Variáveis Sociodemográficas $(\mathrm{n}=64)$

\begin{tabular}{|c|c|c|c|c|c|c|}
\hline & 1 & 2 & 3 & 4 & 5 & 6 \\
\hline 1. Apego materno-fetal & - & - & - & - & - & - \\
\hline \multicolumn{7}{|l|}{ Categorias da entrevista: } \\
\hline 2. Capacidade de ouvir, vocalizar e sorrir & $0,23 *$ & - & - & - & - & - \\
\hline 3. Capacidade de estar alerta e movimentar-se & 0,15 & 0,26 & - & - & - & - \\
\hline 4. Capacidade de demonstrar agrado & $0,26^{*}$ & 0,21 & 0,40 & - & - & - \\
\hline 5. Capacidade de demonstrar desagrado & 0,08 & 0,12 & 0,51 & 0,38 & - & - \\
\hline 6. Capacidade interativa geral & $0,23 *$ & 0,47 & 0,79 & 0,69 & 0,81 & - \\
\hline \multicolumn{7}{|l|}{ Variáveis sociodemográficas: } \\
\hline 7. Idade da gestante & 0,01 & 0,08 & 0,15 & 0,10 & 0,15 & 0,18 \\
\hline 8. Escolaridade da gestante & 0,14 & $-0,07$ & $0,41 * *$ & 0,19 & $0,28 *$ & $0,33 * *$ \\
\hline 9. Renda familiar & 0,01 & $-0,10$ & 0,15 & 0,16 & $0,32 * *$ & $0,23 *$ \\
\hline 10. Número de filhos & $-0,13$ & 0,02 & $-0,15$ & $-0,11$ & $-0,25 *$ & $-0,20$ \\
\hline 11. Idade Gestacional & 0,04 & $0,22 *$ & 0,06 & 0,11 & 0,14 & 0,18 \\
\hline 12. Peso do bebê ao nascer & $-0,04$ & 0,11 & 0,17 & $-0,11$ & 0,05 & 0,08 \\
\hline 13. Comprimento do bebê ao nascer & $-0,13$ & 0,07 & $0,27^{*}$ & $-0,08$ & 0,18 & 0,18 \\
\hline
\end{tabular}

$* \mathrm{p}<0,05 ; * * \mathrm{p}<0,01$. 
Uma análise de regressão stepwise foi realizada com as três variáveis explanatórias que apresentaram correlações significativas com a capacidade interativa geral do bebê. Assim, a escolaridade materna, a renda familiar e o apego materno-fetal foram inseridas como preditoras da percepção das participantes acerca das capacidades interativas dos seus bebês. A Tabela 3 apresenta o resumo dessa análise de regressão. Os valores de beta, os valores de $t$ e níveis de significância são informados para cada um dos modelos testados, assim como os valores da análise de variância e $\mathrm{R}^{2}$ ajustado.

\section{TABELA 3}

Resumo da Análise de Regressão Múltipla (Stepwise) da Percepção Materna Acerca da Capacidade Interativa Geral do Bebê sobre o Apego Materno-fetal, a Escolaridade da Gestante e a Renda familiar $(n=64)$

\begin{tabular}{clccc}
\hline Modelos & \multicolumn{1}{c}{ Variáveis } & $B$ & $t$ & $p$ \\
\hline \multirow{2}{*}{1} & Escolaridade & 0,53 & 2,10 & 0,04 \\
& Renda & 0,001 & 1,22 & 0,22 \\
& Apego Materno-fetal & 0,13 & 1,81 & 0,07 \\
& & $\mathrm{~F}=3,98$ & $p=0,01$ & $\mathrm{R}^{2}=0,13$ \\
\hline \multirow{2}{*}{2} & Escolaridade & 0,62 & 2,47 & 0,01 \\
& Apego Materno-fetal & 0,13 & 1,79 & 0,07 \\
& & $\mathrm{~F}=5,18$ & $p=0,009$ & $\mathrm{R}^{2}=0,12$ \\
\hline
\end{tabular}

Como mostra a Tabela 3, o segundo modelo $(\mathrm{F}=5,18$, $\mathrm{p}=0,009)$ indica que somente o apego materno fetal e a escolaridade materna foram preditores significativos, explicando $12 \%\left(\mathrm{R}^{2}=0,12\right)$ da variância na percepção materna acerca da capacidade interativa geral do bebê. A variável renda familiar foi excluída do modelo. Para cada ponto a mais na escala de apego materno-fetal, a média dos relatos na categoria capacidade interativa geral do bebê aumentou em 0,13 , enquanto a cada ano a mais de escolaridade materna a média dos relatos na categoria capacidade interativa geral do bebê aumentou em 0,62. A análise desses valores beta indicam que a escolaridade materna tende a ser um preditor mais forte da percepção materna acerca da capacidade interativa geral do bebê, se comparado ao apego materno-fetal.

\section{DISCUSSÃO}

Este estudo investigou as relações entre o apego materno-fetal e a percepção da mãe acerca da capacidade interativa do recém-nascido. Os resultados confirmaram que quanto maior o escore de apego materno fetal, maior a frequência de relatos maternos que descrevem as capacidades do bebê de ouvir, vocalizar e sorrir, e de demonstrar agrado e desagrado, assim como a frequência do total de relatos sobre a capacidade interativa geral do bebê durante a entrevista. Essa hipótese também foi corroborada pela análise de regressão que confirmou o poder preditivo do apego materno-fetal sobre a percepção da mãe quanto à capacidade interativa do bebê. A escolaridade materna, que também esteve positivamente correlacionada à variável predita, quando inserida no modelo de regressão, demonstrou um poder preditivo superior aquele revelado pelo apego materno-fetal.

Vários estudos já indicavam o impacto positivo do apego materno fetal sobre o comportamento materno e a interação mãe-bebê após o nascimento da criança (Alvarenga et al., 2013; Mercer \& Ferketich, 1994; Shin et al., 2004; Shin et al., 2006). Os achados do presente estudo revelam que indicadores de uma forte vinculação afetiva da gestante com o feto no último trimestre da gestação predizem maior capacidade de discriminação e de descrição, por parte da mãe, de comportamentos do bebê que indicam seu potencial de comunicação e interação social logo após o nascimento. Considerando a importância dessa capacidade discriminativa no adulto cuidador, para que ele possa estabelecer com a criança padrões de interação sensíveis e responsivos (van den Boom, 1994; Wendland-Carro et al., 1999), e o poder preditivo do apego materno fetal sobre essa característica, os achados deste estudo destacam a relevância de estratégias que detectem problemas e ao mesmo tempo permitam intervenções eficazes para promover o vínculo afetivo entre mãe e feto durante a gestação.

Além da importância dos cuidados com o vínculo afetivo que começa a se estabelecer entre a díade no período pré-natal, os resultados chamam a atenção para o impacto da escolaridade materna sobre a percepção da mãe acerca do potencial interativo do recémnascido. Inúmeros estudos apontam consistentemente para o fato de que pais de baixo nível socioeconômico e com menor escolaridade costumam apresentar mais dificuldades em tarefas ligadas à parentalidade (Alvarenga, Magalhães \& Gomes, 2012; Bordin et al., 2009; Carmo \& Alvarenga, 2012; Pereira, Negrão, Soares, \& Mesman, 2013; Walker et al., 2011). Além desses estudos, a investigação conduzida por Seidlde-Moura et al. (2004) indicou que o fato de as mães avaliarem de forma positiva e acurada as competências do bebê recém-nascido, considerando-o como um ser ativo e participante nas trocas sociais, está relacionado ao seu nível de escolaridade. Essa relação pode ser justificada, pelo menos em parte, por um maior conhecimento sobre o desenvolvimento infantil (Ribas Jr, Seidl-de-Moura \& Bornstein, 2003; Seidl-de-Moura et al., 2004), assim como por uma maior habilidade 
verbal (Zamberlan, 2002), que se refletiriam tanto na percepção mais acurada do comportamento do bebê, como em uma maior capacidade de fazer relatos verbais acerca de tais capacidades. A renda familiar, que também esteve positivamente correlacionada com a percepção da capacidade interativa geral do bebê, mas que foi excluída do modelo de regressão, também foi reportada como um importante preditor de variáveis associadas à parentalidade em outros estudos (Aquino \& Salomão, 2011; Seidl-de-Moura et al., 2004; Ribas Jr et al., 2003). Porém, algumas dessas investigações discutem o fato de que, dentre os fatores que habitualmente são utilizados para avaliar o status socioeconômico, como por exemplo, escolaridade, prestígio ocupacional e renda, a escolaridade parece constituir o componente mais importante a ser considerado (Ribas Jr et al., 2003).

Outras variáveis estiveram relacionadas à percepção da mãe acerca do potencial interativo do recém-nascido, e seus efeitos merecem ser melhor explorados em futuras investigações. Com relação ao número de filhos, o estudo de Dessen e Braz (2000), demonstrou que quanto maior o número de filhos, maior a necessidade de apoio recebido. Já o estudo de Alvarenga et al. (2012), revelou que o maior número de filhos esteve relacionado a menores escores de apego materno-fetal. Juntos, esses estudos podem estar indicando que haveria uma maior demanda sobre a mãe, o que, consequentemente, no contexto investigado no presente estudo, poderia ocasionar uma redução na sua disponibilidade para observar e interpretar os sinais do recém-nascido.

Com relação às variáveis relacionadas à gestação, como a idade gestacional, no presente estudo, as mães de bebês nascidos com maior número de semanas de gestação relataram em média mais capacidades interativas durante a entrevista. É possível que essas relações estejam indicando uma capacidade maior do próprio recém-nascido de demonstrar seu potencial durante a interação com a mãe, seja sorrindo, vocalizando ou protestando com mais frequência e vigor, do que bebês nascidos após uma gestação mais curta. Estudos que compararam bebês nascidos a termo com bebês prematuros, fornecem apoio a essa hipótese, mesmo que de forma indireta, já que a amostra do presente estudo não incluiu bebês prematuros. Esses estudos revelam que os prematuros têm um maior número de alterações no desenvolvimento e um pior desempenho perceptual e motor (Resegue, Puccini, $\&$ da Silva, 2007; Tofail et al., 2012), o que remete à possibilidade de que a menor idade gestacional possa implicar também em diferenças, mesmo que mais sutis, nas capacidades interativas do recém-nascido.
Em resumo, os achados do presente estudo apontam para a importância de serviços disponibilizados à comunidade e estratégias de intervenção em geral, que favoreçam a formação de um vínculo positivo entre mãe e bebê durante a gravidez, estimulando comportamentos de afiliação, interação e cuidados com a criança durante esse período e, portanto, auxiliando a mulher no processo de transição para a maternidade. Outros estudos realizados no Brasil indicam a importância desse tipo de estratégia, seja ao mostrar o impacto de variáveis relacionadas a processos e variáveis psicológicas avaliadas durante a gestação sobre as interações iniciais entre mãe-bebê (Alvarenga et al., 2013), ou ao revelar que as demandas emocionais da gestação não costumam ser contempladas durante a assistência pré-natal (Piccinini, Carvalho, Ourique, \& Lopes, 2012).

O presente estudo tem limitações. Em primeiro lugar, o instrumento que avaliou o apego maternofetal, embora seja amplamente utilizado na literatura (Alhusen, 2008; Yarcheski et al., 2009), não pôde ser explorado em seus diferentes fatores, devido a problemas em suas propriedades psicométricas. Além disso, a percepção das mães acerca das capacidades interativas dos seus bebês foi avaliada apenas por meio de entrevista, sem que procedimentos de observação, que constituem estratégias privilegiadas na avaliação de fenômenos interativos, tenham sido utilizados. Nesse sentido, é importante que futuros estudos investiguem a percepção da mãe acerca das capacidades interativas do bebê através de medidas que possam ser articuladas a procedimentos observacionais. Do mesmo modo, é fundamental que outros instrumentos para a avaliação do apego materno-fetal sejam desenvolvidos, ou que a escala utilizada no presente estudo seja aprimorada, uma vez que o poder preditivo dessa variável tem se mostrado significativo (Alvarenga et al., 2013; Shin et al., 2006). Por fim, é importante lembrar que a amostra do presente estudo foi constituída apenas por mães de bebês do sexo masculino. Assim, seria interessante que futuras investigações incluíssem bebês de ambos os sexos para que eventuais diferenças no potencial interativo e no seu impacto sobre a percepção materna pudessem ser avaliados.

\section{CONSIDERAÇÕES FINAIS}

$\mathrm{O}$ presente estudo demonstrou que o apego materno-fetal é um preditor significativo da percepção materna acerca das capacidades interativas do bebê. A escolaridade materna destacou-se no modelo de predição, apontando para a importância desse fator sociodemográfico, e reforçando a noção de maior 
vulnerabilidade das populações pobres e desassistidas no que se refere às tarefas relacionadas à parentalidade.

A relação que a mãe estabelece com o seu bebê, desde a gravidez, é um importante preditor das características da futura interação mãe-criança. Sendo assim, estudos como este, que investigam variáveis típicas do período pré-natal, e do processo de transição para a maternidade que ocorre ao longo da gestação, são extremamente relevantes, porque podem contribuir com a identificação de fatores de risco e com a redução do seu impacto negativo sobre a interação da díade e sobre o desenvolvimento infantil.

\section{REFERÊNCIAS}

Abbad, G. \& Torres, C. V. (2002). Regressão múltipla stepwise e hierárquica em Psicologia Organizacional: aplicações, problemas e soluções. Estudos de Psicologia (Natal), 7(n. esp.), 19-29. http://dx.doi.org/10.1590/S1413294X2002000300004

Alhusen, J. L. (2008). A Literature update on maternal-fetal attachment. Journal of Obstetric, Gynecologic, \& Neonatal Nursing, 37, 315-328. http://dx.doi.org/10.1111/j.1552-6909.2008.00241.x

Alhusen, J. L., Gross, D., Hayat, M. J., Woods, A. B., \& Sharps, P. W. (2012). The influence of maternal-fetal attachment and health practices on neonatal outcomes in low-income, urban women. Research in Nursing \& Health, 35(2), 112-120. http://dx.doi.org/10.1002/nur.21464

Alvarenga, P., Dazzani, M. V. M., Alfaya, C. A. S., Lordelo, E. R., \& Piccinini, C. A. (2012). Relações entre a saúde mental da gestante e o apego materno-fetal. Estudos de Psicologia (Natal), 17(3), 477-484. http://dx.doi.org/10.1590/ S1413-294X2012000300017

Alvarenga, P., Magalhães, M. O., \& Gomes, Q. S. (2012). Relações entre práticas educativas maternas e problemas de externalização em pré-escolares. Estudos de Psicologia (Campinas), 29(1), 33-42. http://dx.doi.org/10.1590/S0103166X2012000100004

Alvarenga, P., Dazzani, M. V. M., Alfaya, C. A. S., Lordelo, E. R., \& Piccinini, C. A. (2013). Predictors of sensitivity in mothers of 8-month-old infants. Paidéia (Ribeirão Preto), 56(23), 311-319. http://dx.doi.org/10.1590/198243272356201305

Aquino, F. de S. B., \& Salomão, N. M. R. (2011). Percepções maternas acerca das habilidades sociocomunicativas de bebês. Psicologia: Ciência e Profissão, 31(2), 252-267. http://dx.doi.org/10.1590/S1414-98932011000200005

Bardin, L. (2011). Análise de Conteúdo. São Paulo: Edições 70.

Bordin, I. A., Duarte, C. S., Peres, C. A., Nascimento, R., Curto, B. M., \& Paula, C. S. (2009). Severe physical punishment: risk of mental health problems for poor urban children in Brazil. Bulletin World Health Organization, 87(5), 336-344. http://dx.doi.org/10.2471/BLT.07.043125

Bowlby, J. (2002). Apego e perda: apego (3ㅜㅜ ed.) (Vol. 1). São Paulo: Martins Fontes.

Brazelton, T. B. (1988). O desenvolvimento do apego: uma família em formação. Porto Alegre: Artes Médicas.

Carmo, P. H. B. \& Alvarenga, P. (2012). Práticas educativas coercitivas de mães de diferentes níveis socioeconômicos. Estudos de Psicologia, 17(2), 191-197. http://dx.doi.org/10.1590/S1413-294X2012000200001

Cerezo, M. A., Trenado, R. M., \& Pons-Salvador, G. (2012). Mother-infant interaction and quality of child's attachment: a nonlinear dynamical systems approach. Nonlinear Dynamics, Psychology, and Life Sciences, 16(3), 243-267. http:// dx.doi.org/10.1016/j.infbeh.2008.07.010

Costa, R. \& Figueiredo, B. (2012). Infants psychophysiological profile and mother-infant interaction. International Journal of Behavioral Development, 36(3), 205-214. http://dx.doi.org/10.1177/0165025411428248

Cranley, M. S. (1981). Development of tool for measurement of maternal attachment during pregnancy. Nursing Research, 30(5), 281-284. http://dx.doi.org/10.1097/00006199-198109000-00008

Dancey, C. P., \& Reidy, J. (2013). Estatística sem matemática para psicólogos. Porto Alegre: Penso.

Dessen, M. A., \& Braz, M. P. (2000). Rede social de apoio durante transições familiares decorrentes do nascimento de filhos. Psicologia: Teoria e Pesquisa, 16(3), 221-231. http://dx.doi.org/10.1590/S0102-37722000000300005

Doan, H. M., Cox, N. L., \& Zimmerman, A. (2003). The maternal fetal attachment scale: some methodological ponderings. Journal of Prenatal and Perinatal Psychology and Health, 18, 167-188.

Emerich, D. R., da Rocha, M. M., Silvares, E. F. de M., \& Gonçalves, J. de P. (2012). Diferenças quanto ao gênero entre escolares brasileiros avaliados pelo inventário de comportamentos para crianças e adolescentes (CBCL/6-18). Psico (Porto Alegre), 43(3), 380-387. http://revistaseletronicas.pucrs.br/ojs/index.php/revistapsico/article/view/10053/8239

Fearon, R. P., Bakermans-Kranenburg, M. J., van IJzendoorn, Lapsley, M. H., \& Roisman, G. I. (2010). The significance of insecure attachment and disorganization in the development of children's externalizing behavior: a meta-analytic study. Child Development, 81(2), 435-456. http://dx.doi.org/10.1111/j.1467-8624.2009.01405.x

Feijó, M. C. C. (1999). Validaçäo brasileira da "maternal-fetal attachment scale". Arquivos Brasileiros de Psicologia, 51(4), 52-62. 
Fraley, R. C., Roisman, G. I., \& Haltigan, J. D. (2013). The legacy of early experiences in development: formalizing alternative models of how early experiences are carried forward over time. Developmental Psychology, 49(1), 109-126. http://dx.doi.org/10.1037/a0027852

Klaus, M. \& Kennel, J. (1992). Pais/bebê: a formação do apego. Porto Alegre: Artes Médicas.

Loo, K. K., Ohgi, S., Howard, J., Tyler, R., \& Hirose, T. (2005). Neurobehaviors of Japanese newborns in relation to the characteristics of early mother-infant interaction. The Journal of Genetic Psychology, 166(3), 264-279. http://dx.doi. org/10.3200/GNTP.166.3.264-279

Mercer, R. T. \& Ferketich, S. L. (1994). Predictors of maternal role competence by risk status. Nursing Research, 43(1), 38-43. http://dx.doi.org/10.1097/00006199-199401000-00009

Núcleo de Infância e Família - NUDIF/UFRGS/CNPq. (2006). Experiência da Maternidade e o Desenvolvimento do Segundo Filho aos 12 meses. (Instrumento não publicado). Instituto de Psicologia. Universidade Federal do Rio Grande do Sul.

Pereira, M., Negrão, M., Soares, I., \& Mesman, J. (2013). Predicting harsh discipline in at-risk mothers: the moderating effect of socioeconomic deprivation severity. Journal of Child and Family Studies, 1-9 http://dx.doi.org/10.1007/ sf0826-013-9883-2

Piccinini, C. A., Gomes, A. G., Nardi, T., \& Lopes, R. S. (2008). Gestação e a constituição da maternidade. Psicologia em Estudo (Maringá), 13(1), 63-72. http://dx.doi.org/10.1590/S1413-73722008000100008

Piccinini, C. A., Carvalho, F. T., Ourique, L. R., \& Lopes, R. S. (2012). Percepções e sentimentos de gestantes sobre o pré-natal. Psicologia: Teoria e Pesquisa, 28(1), 27-33. http://dx.doi.org/10.1590/S0102-37722012000100004

Pisoni, C., Garofoli, F., Tzialla, C., Orcesi, S., Spinillo, A., Politi, P., Balottin, U., Manzoni, P., \& Stronati, M. (2014). Risk and protective factors in maternal-fetal attachment development. Early Human Development, 90S2, S45-S46. http://dx.doi.org/10.1016/S0378-3782(14)50012-6

Resegue, R., Puccini, R. F., \& da Silva, E. M. K. (2007). Fatores de risco associados a alterações no desenvolvimento da criança. Pediatria (São Paulo), 29(2), 117-128. http://dx.doi.org/10.1590/S1516-31802008000100002

Ribas Jr, R. C., Seidl de Moura, M. L., Soares, I. D., Gomes, A. A. N., \& Bornstein, M. H. (2003). Socioeconomic status in Brazilian psychological research: I. validity, measurement, and application. Estudos de Psicologia, 8(3), 375-383. http://dx.doi.org/10.1590/S1413-294X2003000300004

Schwengber, D. D. S., \& Piccinini, C. A. (2005). A experiência da maternidade no contexto da depressão materna no final do primeiro ano de vida do bebê. Estudos de Psicologia (Campinas), 22, 143-156. http://dx.doi.org/10.1590/ S0103-166X2005000200004

Seidl de Moura, M. L., Ribas, A. F. P., Seabra, K. da C., Pessôa, L. F., Ribas Jr, R. de C., \& Nogueira, S. E. (2004). Interações iniciais mãe-bebê. Psicologia: Reflexão e Crítica, 17(3), 295-302. http://dx.doi.org/10.1590/S010279722004000300002

Shieh, C., Kravitz, M., \& Wang, H. (2001). What do we know about maternal-fetal attachment? The Kaohsiung. Journal of Medicinal Sciences, 17, 448-454.

Shin, H. J., Park, Y. J., \& Kang, H. C. (2004). Prediction model on mother-infant attachment during the early postpartum period. Taehan Kanho Hakhoe Chi, 34(3), 504-514.

Shin, H., Park, Y. J., \& Kim, M. J. (2006). Predictors of maternal sensitivity during the early postpartum period. Journal of Advanced Nursing, 55(4), 425-434. http://dx.doi.org/10.1111/j.1365-2648.2006.03943.x

Taffazoli, M., Asadi, M. M., Aminyazdi, S. A., \& Shakeri, M. T. (2015). The relationship between maternal-fetal attachment and mother-infant attachment behaviors in primiparous women referring to Mashhad health centers. Journal of Midwifery \& Reproductive Health, 3(2), 318-327.

Tofail, F., Hamadani, J. D., Ahamed, A. Z. T., Mehrin, F., Hakim, M., \& Huda, S. N. (2012). The mental development and behavior of low-birth-weight Bangladeshi infants from urban low-income community. European Journal of Clinical Nutrition, 66, 237-243. http://dx.doi.org/10.1038/ejcn.2011.165

Vandell, D. L., Belsky, J., Burchinal, M., Steinberg, L., \& Vandergrift, N. (2010). Do effects of early child care extend to age 15 years? Results from the NICHD Study of Early Child Care and Youth Development. Child Development, 81(3), 737-756. http://dx.doi.org/10.1111/j.1467-8624.2010.01431.x

Van den Boom, D. C. (1994), The influence of temperament and mothering on attachment and exploration: an experimental manipulation of sensitive responsiveness among lower-class mothers with irritable infants. Child Development, 65, 1457-1477. http://dx.doi.org/10.1111/j.1467-8624.1994.tb00829.x

Yarcheski, A., Mahon, N. E., Yarcheski, T. J., Hanks, M. M., \& Cannella, B. L. (2009). A meta-analytic study of predictors of maternal-fetal attachment. International Journal of Nursing Studies, 46(5), 708-715. http://dx.doi.org/10.1016/j. ijnurstu.2008.10.013

Walker, S. P., Wachs, T. D, Grantham-McGregor, S., Black, M. M., Nelson, C. A., Huffman, S. L., Baker-Henningham, H., Chang, S. M., Hamadani, J. D., Lozoff, B., Gardner, J. M. M., Powell, C. A., Rahman, A., \& Richter, L. (2011) Inequality in early childhood: risk and protective factors for early child development. The Lancet, 378, 1325-1338. http://dx.doi.org/10.1016/S0140-6736(11)60555-2

Wendland, J. \& Piccinini, C. A. (1998). Effets d'une intervention précoce visant à favoriser la qualité de l'interaction mère-bébé. Psychiat Enfant, 41(1), 187-217. 
Wendland-Carro, J., Piccinini, C. A., \& Millar, W. S. (1999) The role of early intervention on enhancing the quality of mother-infant interaction. Child Development, 70(3), 713-721. http://dx.doi.org/10.1111/1467-8624.00051

Zamberlan, M. A. T. (2002). Interação mãe-criança: enfoques teóricos e implicações decorrentes de estudos empíricos. Estudos de Psicologia, 7(2), 399-406. http://dx.doi.org/10.1590/S1413-294X2002000200021

Zhu, H., Loo, K.K., Min, L., Yin, Q., Luo, H., Chen, L. et al. (2007). Relationship between neurobehaviours of Chinese neonates and early mother-infant interaction. Journal of Reproductive and Infant Psychology, 25(2), 106-121. http:// dx.doi.org/10.1080/02646830701292340

Autores:

PATRícia ALVARENGA - Doutora, Universidade Federal da Bahia.

JANAÍNA NASCIMENTO TEIXEIRA - Mestre, Universidade Federal da Bahia.

Aline Carvalho PeiXoto - Mestre, Universidade Federal da Bahia.

\section{Endereço para correspondência:}

Patrícia Alvarenga

Rua Aristides Novis, 197

CEP 40210-730 Salvador, BA, Brasil

Recebido em: 22.09.14

Aceito em: 28.05.15 